\title{
Efeito de Sedimentos Contaminados Sobre a Excreção de Nitrogênio do Camarão Penaeus paulensis
}

\author{
Rosane B.C. Moraes ${ }^{1 *}$, Wolfgang C. Pfeiffer ${ }^{2}$, Jean R. Guimarães ${ }^{2}$, Alexandre Luiz N. \\ Borges $^{1}$ and Alexandre N. Campos ${ }^{1}$. \\ ${ }^{I}$ Departamento de Biologia Marinha - Instituto de Biologia - Universidade Federal Fluminense - Caixa Postal \\ 100.644, CEP 24001-970, Niterói - RJ, Brasil; ${ }^{2}$ Laboratório de Radioisópotos - Instituto de Biofísica - \\ Universidade Federal do Rio de Janeiro.
}

\begin{abstract}
Physiological response (ammonia excretion) of the shrimp Penaeus paulensis exposed to contaminated sediments of Guanabara Bay and Sepetiba Bay (Rio de Janeiro - Brazil), was monitored for 52 days. The toxicity tests were conducted in aquarium with a water feedback system and a sediment layer of $2 \mathrm{~cm}$. The sediment from Saco do Engenho (Sepetiba Bay) was the most toxic, affecting the excretion of P. paulensis. In sediment from Sepetiba Bay the ammonia excretion was significantly enhanced after 10 days (S. Engenho) and 52 days (I. Madeira) of exposition.
\end{abstract}

Key words: sediment, toxicity, excretion, Penaeus.

\section{INTRODUÇÃO}

Contaminantes tóxicos podem estar contribuindo para o declínio de certas populações de camarões em algumas regiões estuarinas. Os níveis de contaminantes dissolvidos em muitos ambientes estuarinos estão frequentemente abaixo dos níveis conhecidos que causam efeito agudo. Entretanto, efeitos biológicos sub-letais podem estar ocorrendo nos organismos em resposta aos níveis baixos de exposição ao contaminante.

Os resultados de estudos de efeitos subletais e crônicos frequentemente permitem predizer $o$ potencial do impacto ambiental de um poluente. Segundo Bayne et al. (1985), alterações na condição fisiológica de organismos podem ser uma indicação de alerta de estresse uma vez que representam uma integração dos efeitos bioquímicos e citológicos que podem se alterar em resposta a mudanças no ambiente. Respostas fisiológicas podem ser capazes de refletir a deterioração do ambiente antes dos efeitos se manifestarem a nível de população ou de comunidade.
Camarões do gênero Penaeus ocorrem ao longo da costa brasileira em estuários, lagoas costeiras, baías e em mar aberto (Silva, 1977) e representam uma significativa fração da produção pesqueira nacional. A partir do estágio de desenvolvimento denominado "misis", esses organismos assumem hábitos bentônicos e detritívoros (I.N.E.M, 1985) colocando-os em constante contato com o sedimento que é o compartimento ambiental de maior retenção de poluentes. Cripe e Cripe (1990) apontam os peneídeos como uma família de crustáceos estuarinos de grande sensibilidade a materiais tóxicos.

A avaliação da sensibilidade de pós-larvas de peneídeos a contaminantes, pode prover um nível de referência para permitir a proteção desta espécie mediante a regulamentação do despejo de contaminantes. A excreção é uma função fisiológica essencial responsável pela eliminação de resíduos metabólicos tóxicos. $\mathrm{O}$ nitrogênio, como produto de excreção, é derivado do catabolismo de proteínas e sua concentração é determinada pela utilização de proteínas para energia e pela taxa de cisão e

\footnotetext{
* Autor para correspondência
} 
"turnover" de constituintes celulares. Invertebrados aquáticos excretam a maior parte de seu nitrogênio na forma de amônia e a proporção de nitrogênio como produto de excreção pode variar com a estação do ano e como um resultado de estresse ambiental (Bayne et al., 1985).

O objetivo deste trabalho foi avaliar o efeito de sedimentos de ambientes contaminados na excreção de pós-larvas do camarão Penaeus paulensis. Uma vez que a poluição ambiental vem aumentando nas áreas tropicais, estudos ecotoxicológicos com espécies nativas são necessários para o desenvolvimento de um eficiente programa de monitoramento ambiental.

\section{MATERIAL E MÉTODOS}

Sedimentos foram colhidos em três baías do Estado do Rio de Janeiro: Baía de Guanabara, Baía de Sepetiba e Baía da Ilha Grande (Fig. 1). As regiões estudadas nas baías de Guanabara e de Sepetiba foram aquelas apontadas na literatura como áreas de elevada contaminação por substâncias orgânicas ou inorgânicas.

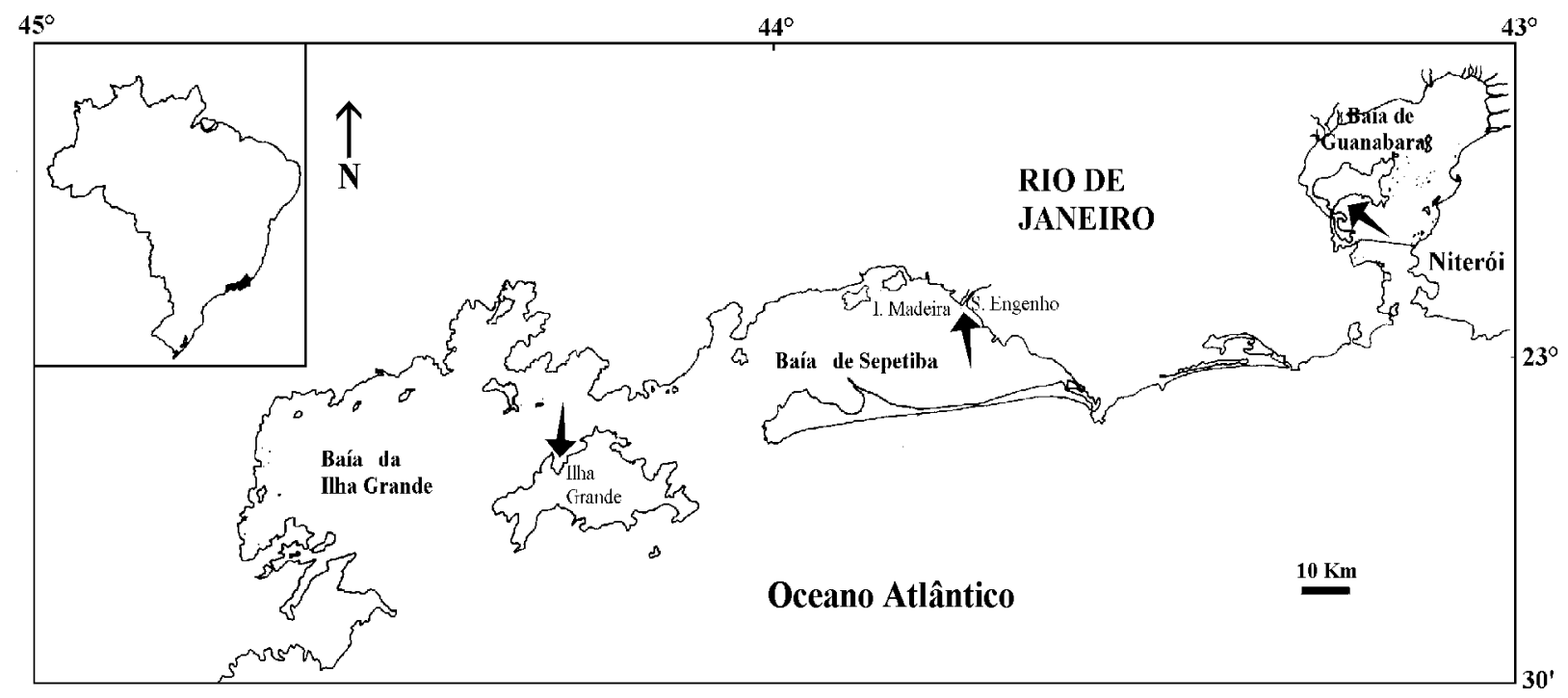

Figura 1. Localização das áreas de colheita de sedimentos

O sedimento da Baía da Ilha Grande foi utilizado como controle, uma vez que a região é relativamente distante de grandes centros urbanos e industriais.

A colheita do sedimento foi feita utilizando-se uma draga de "Ekman" de aço inox. Somente a camada superior do sedimento $(2 \mathrm{~cm})$ foi colhida com uma colher de teflon, condicionada em sacos plásticos e transportada para o laboratório em isopor com gelo. No laboratório, cada sedimento foi homogeinizado com uma colher de teflon até a coloração e a textura estarem visualmente uniformes. Sub-amostras de cada tipo de sedimento foram separadas e estocadas em congelador para posterior análise do teor de matéria orgânica e de metais pesados. $O$ teor de matéria orgânica foi determinado por diferença de peso entre o sedimento seco e o sedimento calcinado $\left(550^{\circ} \mathrm{C}\right)$ e os metais fracamente ligados ao sedimento, foram extraídos por lixiviação com $\mathrm{HCl} 0,1 \mathrm{~N}$ (16 h) e quantificados por espectrofotometria de absorção atômica (equipamento Varian AA 1475). O tamanho do grão do sedimento foi determinado em sub-amostras através de técnica padrão de peneiramento. Todos os sedimentos amostrados possuíam granulometria fina $(<63$ $\mu \mathrm{m})$. Uma camada de $2 \mathrm{~cm}$ de cada sedimento homogeinizado foi adicionada em aquários de vidro $(20$ X $20 \times 20 \mathrm{~cm})$ e coberta com 5 litros de água do mar sem ressuspender o sedimento. 
A água do mar foi colhida na Praia do Recreio dos Bandeirantes (Rio de Janeiro) e ajustada para salinidade 30 com adição de água destilada. Em cada aquário foi introduzido um pequeno filtro biológico, constituído de fragmentos de conchas e pedras dolomíticas, com a finalidade de manter baixas as concentrações dos compostos nitrogenados tóxicos (amônia e nitrito). Cada tipo de sedimento foi testado em triplicata.

Pós-larvas do camarão Penaeus paulensis foram obtidas do Laboratório de Larvicultura de Peneídeos da Universidade Federal de Santa Catarina e aclimatadas em aquários por aproximadamente 10 dias. Durante a aclimatação e o período experimental, os animais foram alimentados com ração para camarão desenvolvida no Laboratório de Larvicultura.

Após o período de aclimatação, vinte pós-larvas (9 a $13 \mathrm{~mm}$ de comprimento total) foram introduzidas em cada aquário teste. Os animais foram mantidos à temperatura de $22 \pm 1^{\circ} \mathrm{C}$, fotoperíodo de $10 \mathrm{~h}$ de luz e alimentação a cada dois dias. A qualidade da água nos aquários foi monitorada uma vez por semana e consistia de medidas de $\mathrm{pH}$, salinidade, oxigênio dissolvido (OD), amônia e nitrito. O período de exposição dos animais foi de 10,28 e 52 dias.

Após cada período de exposição, 6 animais de cada aquário foram colocados separadamente em frascos de vidro de $250 \mathrm{ml}$ com $200 \mathrm{ml}$ de água do mar $(S=30)$. Os frascos foram devidamente vedados e após 48 horas foram retiradas amostras da água para a determinação da concentração de amônia $\left(\mathrm{N}-\mathrm{NH}_{3}\right)$ pelo método do indofenol (Parsons et al., 1984). A taxa de excreção de cada organismo foi determinada subtraindo-se a concentração de nitrogênio amoniacal da água do frasco controle (sem animal) pela concentração de $\mathrm{N}^{-\mathrm{NH}_{3}}$ da água dos frascos com animais. Ao término do período de excreção, foi determinado o peso seco de cada organismo e os resultados de excreção foram expressos em $\mathrm{mg}$ de $\mathrm{N}-\mathrm{NH}_{3} / \mathrm{g}$ peso seco/h.

A análise de variância (ANOVA) e o Teste de Variação Múltipla de Ducan foram utilizados para verificar a existência de diferenças significativas na excreção dos organismos submetidos aos sedimentos testes.

\section{RESULTADOS}

Os resultados mostram uma elevação na taxa de excreção dos animais expostos aos sedimentos contaminados em relação aos do sedimento da Ilha Grande (controle). As diferenças observadas na taxa de excreção de animais expostos ao mesmo tipo de sedimento ao longo do tempo, devem-se à variação da taxa metabólica do animal com o crescimento. A excreção dos animais expostos aos sedimentos do Saco do Engenho e da Ilha da Madeira apresentaram diferenças estatisticamente significantes $(\mathrm{P} \leq 0,05)$ em relação aos animais controles. A alteração da taxa de excreção é mais marcante após 10 dias e após 52 dias de exposição (Fig. 2). Após 10 dias de exposição, $P$. paulensis apresentou significativa elevação na taxa de excreção quando exposto ao sedimento da Ilha da Madeira. Entretanto, na continuidade da exposição os níveis retornaram a valores próximos aos dos animais controles.

\section{DISCUSSÃO}

A elevação da taxa de excreção pode ser um mecanismo pelo qual o organismo tenta eliminar o composto tóxico do seu interior. Segundo Bayne et al. (1985), a proporção de nitrogênio como produto de excreção pode variar como resultado do estresse ambiental. A resposta fisiológica pode ser iniciada como um meio do animal manter a homeostase ou pode ser reflexo da alteração de alguma função (Heath, 1987). 


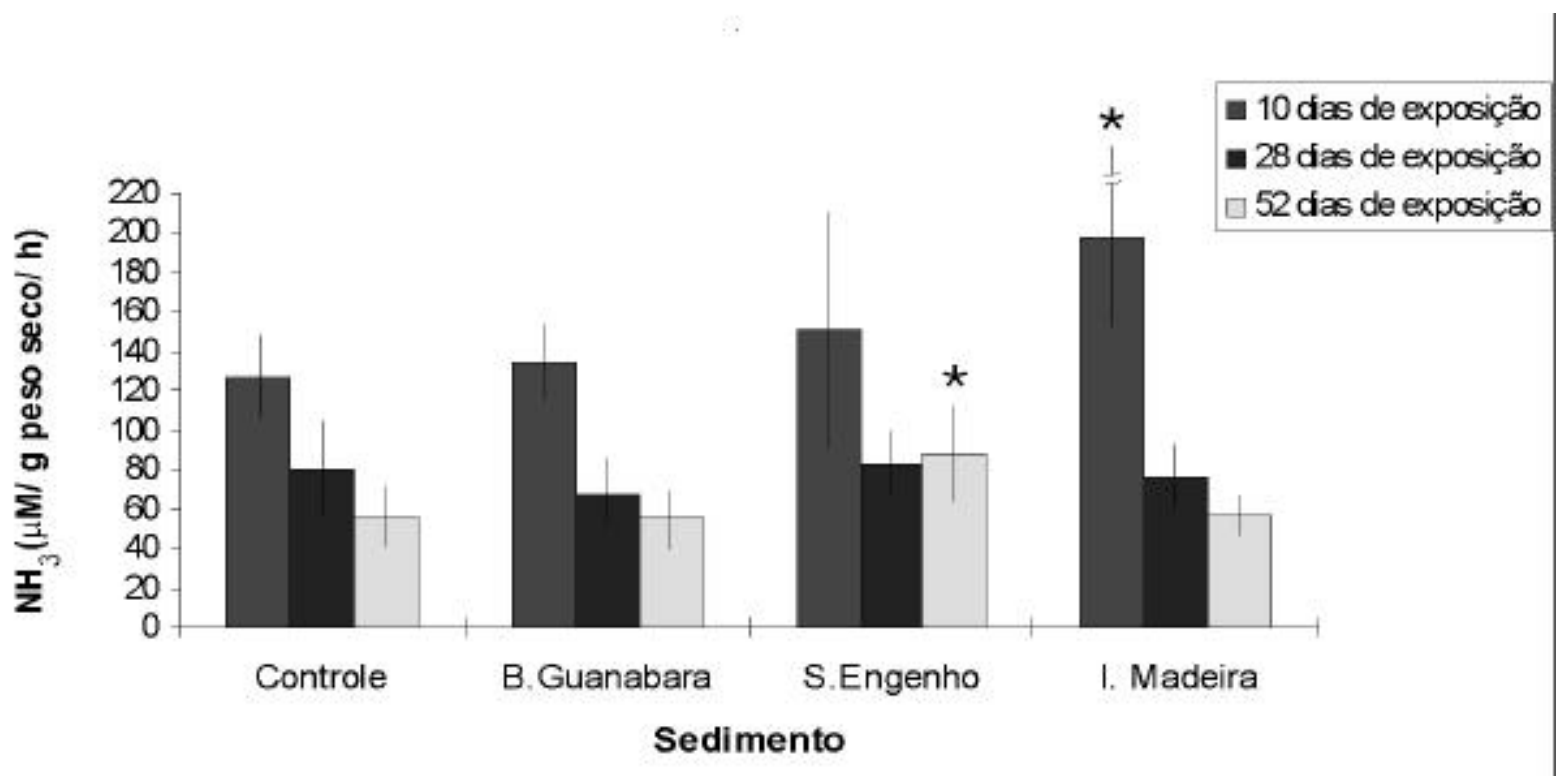

Figura 2. Taxa de excreção de amônia de pós-larvas de $P$. paulensis após cada período de exposição a diferentes sedimentos. Cada barra representa o valor médio de três réplicas e a linha vertical, o desvio padrão. * Indica diferença significativa $(\mathrm{p} \leq 0,05)$ entre o tratamento e o controle.

A compensação fisiológica, frequentemente chamada de aclimatação, é um fenômeno que ocorre em muitos organismos. Inicialmente, uma exposição a um fator ambiental alterado (Ex.: temperatura, poluente químico) levará o organismo a exibir alguma resposta fisiológica. Entretanto, sob uma exposição contínua, se o grau de estresse é leve, o fator fisiológico pode exibir compensação (aclimatação) e tender ao retorno à condição inicial isto é, de animal não exposto (Heath, 1987). A compensação fisiológica pode ser observada nas pós-larvas de $P$. paulensis expostas ao sedimento da Ilha da Madeira, revelando que este sedimento ainda apresenta um grau de contaminação no qual a espécie é capaz de se adaptar.

Elevação e diminuição da taxa de excreção têm sido observadas em organismos aquáticos expostos a diferentes contaminantes. $\mathrm{O}$ aumento da excreção de amônia foi verificado em mexilhões expostos a cobre (Moore et al., 1984; Krishnakumar et al., 1990) e a mercúrio (Krishnakumar et al., 1990).
O efeito do cádmio como depressor da taxa de excreção foi observado no mexilhão Lampsilis ventricosa (Naimo et al., 1992) e no misidáceo Leptomysis lingvura (Gaudy et al., 1991). Laughlin e Lindén (1983) obtiveram diminuição da excreção de amônia em misidáceos, Neomysis integer, após exposição à fração hidrosolúvel de um óleo refinado. Moraes et al. (1994) também verificaram que camarões da espécie Penaeus brasiliensis expostos a $10 \mathrm{mg}$ Zn..$^{-1}$ por $96 \mathrm{~h}$ diminuíram a taxa de excreção de amônia.

A variação da resposta na taxa de excreção parece ser dependente do tempo de exposição. Cheung e Cheung (1995) relataram que mexilhões da espécie Perna viridis, expostos a cádmio e zinco, exibiram, inicialmente, um aumento da taxa de excreção seguido de um decréscimo em relação aos indivíduos controle.

O aumento da excreção de amônia é indicativo de aumento de catabolismo proteico (Moore $e t$ al., 1984). A concentração de excretas 
nitrogenados é determinada pela utilização de proteínas como fonte de energia e pela taxa de utilização e de "turnover" de constituintes celulares (Bayne et al., 1985). A elevação da taxa de excreção observada em $P$. paulensis parece refletir um aumento da demanda energética do animal na tentativa de excretar as substâncias tóxicas.
O Saco do Engenho apresenta uma elevada contaminação por $\mathrm{Mn}$ e $\mathrm{Zn}$ (Tab.I) em decorrência de sua proximidade com uma indústria metalúrgica, responsável também pela contaminação de arsênico (Magalhães \& Pfeiffer, 1995) na mesma região.

Tabela 1. Características químicas do sedimento das áreas de estudo.

\begin{tabular}{lccccccccc}
\hline & \multicolumn{7}{c}{ Metais } & \multicolumn{7}{c}{$(\mu \mathrm{g} / \mathrm{g}$ sedimento } \\
& $\mathrm{seco})$ & & \\
\hline & $\mathrm{Cd}$ & $\mathrm{Cr}$ & $\mathrm{Cu}$ & $\mathrm{Fe}$ & $\mathrm{Mn}$ & $\mathrm{Ni}$ & $\mathrm{Zn}$ & $\mathrm{Pb}$ & $\%$ MO. \\
\hline Controle & $<0,003$ & 0,26 & $<0,12$ & 18 & 5 & 0,85 & 1 & 0,25 & 15 \\
B. Guanabara & 0,79 & 0,50 & 0,46 & 39 & 75 & 2,42 & 49 & 0,77 & 17 \\
I. Madeira & 3,10 & 1,03 & 0,50 & 518 & 23 & 1,56 & 576 & 2,25 & 13 \\
S. Engenho & 1,90 & 0,25 & 0,14 & 112 & 271 & 1,57 & 5030 & 0,40 & 13 \\
\hline
\end{tabular}

MO - matéria orgânica

O efeito tóxico do zinco em organismos aquáticos tem sido bastante documentado. A letalidade aguda de sais de zinco a peixes marinhos e estuarinos tem sido mostrada ser espécie específica e variar de 100 a $200 \mathrm{mgZn} / \mathrm{l}$ (Eisler \& Hennekey, 1977; Crespo \& Balasch, 1980). A elevada sensibilidade de crustáceos ao zinco foi verificada por Mc Kenney \& Neff (1981) que observaram redução da percentagem de sobrevivência em pós-larvas do camarão Palaemonetes pugio exposto a $0,25 \mathrm{mgZn} / \mathrm{l}$. Resultados comparativos de mortalidade em 48 horas, indicam que o zinco é o metal mais tóxico para Penaeus brasiliensis (Chung, 1980).

O efeito de metais na excreção de organismos aquáticos mostra-se contraditório e parece dependente da espécie, do metal utilizado e do período de exposição. Gaudy (1983) observou diminuição na excreção de amônia quando o misidáceo Leptomysis lingvura foi exposto ao cádmio. Resultados semelhantes foram observados por Naimo et al. (1992) expondo o molusco Lampsilis ventricosa ao cádmio e por Moraes et al. (1994) expondo o camarão Penaeus brasiliensis a diferentes concentrações de zinco.

No presente trabalho, pós-larvas de Penaeus paulensis aumentaram a taxa de excreção quando expostas aos sedimentos contaminados.
White e Rainbow (1982) mostraram a habilidade de crustáceos na regulação de metais pesados como uma resposta adaptativa à elevação das concentrações externas. Embora esta resposta fisiológica possa ser interpretada como um simples mecanismo de adaptação do organismo, o extenso período no qual o organismo mantêm elevado seu metabolismo leva-o a um enfraquecimento, deixando-o mais suscetível a doenças e à predação. Os dados de excreção associados com a redução da sobrevivência observada por Moraes (1996) nas amostras de sedimento contaminado corroboram com esta afirmação. Segundo Amiard et al. (1987), a própria regulação pode ser considerada como um distúrbio no metabolismo normal desde que a perda da estabilidade da concentração interna do metal, por aumento da concentração externa, necessita de uma aceleração de fluxo (White \& Rainbow, 1982) e uma elevada descarga de energia.

\section{CONCLUSÕES}

Apesar dos efeitos observados estarem provavelmente associados à contaminação por metais pesados, não pode ser descartada a hipótese do efeito ser devido à presença de outras substâncias tóxicas não analisadas neste 
trabalho, agindo isoladamente ou em conjunto com os metais.

Embora a concentração de metais disponíveis não apresente valores muito elevados na Baía de Guanabara (Tab.I), esta região é considerada poluída com elevada carga orgânica e presença de várias substâncias oriundas de indústrias químicas e refinarias de petróleo (FEEMA, 1991). A baixa toxicidade desse sedimento após o longo período de exposição pode significar que os compostos tóxicos não estão disponíveis para a biota ou que as substâncias tóxicas ali presentes agem muito lentamente. Experimentos de toxicidade com sedimentos de outros pontos da baía e com um maior período de exposição, devem ser realizados para uma melhor avaliação.

Considerando que $P$. paulensis é um recurso pesqueiro de grande importância econômica, a avaliação da sensibilidade desta espécie à contaminação ambiental, fornece subsídios importantes para a manutenção e a recuperação dos estoques das populações naturais.

Agradecimentos: Este trabalho teve o apoio de: UFRJ, PRONEX/ MCT e FAPERJ.

\section{REFERÊNCIAS}

Amiard, J.C.; Amiard-Triquet, C.; Berthet, B. \& Metayer C. (1987), Comparative study of the patterns of bioaccumulation of essential $(\mathrm{Cu}, \mathrm{Zn})$ and non-essential $(\mathrm{Cd}, \mathrm{Pb})$ trace metals in various estuarine and coastal organisms. J. of Exp. Mar. Biol. and Ecol., 106: $73-89$.

Bayne, B.L.; Brown, D.A.; Burns, K.; Dixon, D.R.; Ivanovici, A.; Lowe, D.M.; Moore, M.N.; Stebbing, A.R.D. \& Widdows, J.(1985), The Effects of Stress and Pollution on Marine Animals. New York: Praeger Scientific. pp.65-89

Cheung, S.G \& Cheung, R.Y.H. (1995), Effects of heavy metals on oxygen consumption and ammonia excretion in green-lipped mussels (Perna viridis). Mar. Pollut. Bull., 31: 381386.
Chung, K.S. (1980), The acute toxicity of four heavy metals $\left(\mathrm{Cd}^{++}, \mathrm{Cr}^{++}, \mathrm{Cu}^{++}\right.$and $\left.\mathrm{Zn}^{++}\right)$to the juvenile spotted brown shrimp (Penaeus brasiliensis). Boletim do Instituto Oceanográfico de São Paulo, 29: 101-106.

Crespo, S. \& Balasch, J.(1980), Mortality, accumulation and distribution of $\mathrm{Zn}$ in the gill system of the dogfish following zinc treatment. Bulletin Environ. Contam. Toxicol., 24: 940-944.

Cripe, G.M. \& Cripe, C.R. (1990), Comparative acute sensitivity of selected estuarine and marine crustaceans to toxic substances. EPA 600/x-90/358. U.S. Environmental Protection Agency, Gulf Breeze, FL.

Eisler, R. \& Hennekey, R.J. (1977). Acute toxicities of $\mathrm{Cd}^{+2}, \mathrm{Cr}^{+6}, \mathrm{Hg}^{+2}, \mathrm{Ni}^{+2}$ and $\mathrm{Zn}{ }^{+2}$ to estuarine macrofauna. Arch. Environ. Contam. Toxicol., 6: 315- 323.

FEEMA. (1991), Qualidade ambiental do ecossistema da bacia hidrográfica da Baía de Guanabara em 1990. FEEMA, Rio de Janeiro, pp. 9-26.

Gaudy, R., J.-P.Guérin \& Kerambrun, P. (1991), Sublethal effects of cadmium on respiratory metabolism, nutrition, excretion and hydrolase activity in Leptomysis lingvura (crustacea: mysidacea). Mar. Biol., 109: 493501.

Gaudy, R. (1983), Action des métaux lourds sur le métabolisme (respiration, excrétion), la nutrition et la reproduction des crustacés. Oceanis. 9: 613-625.

Heath, A.G. (1987), Water Pollution and Fish Physiology. Boston: CRC Press, pp.24-45

I.N.E.M (INSTITUTO NACIONAL DE ESTUDOS DO MAR), 1985, Manual de Maricultura. Ministério da Marinha. pp.2328

Krishnakumar, P.K.; Asokan, P.K \& Pillai, V.K. (1990). Phisiological and cellular responses to copper and mercury in the green mussel Perna viridis (Linnaeus). Aquat.Toxicol., 18: 163-174.

Laughlin, R. \& Lindén, O. (1983), Oil pollution and Baltic mysids: acute and chronic effects of the water soluble fractions of light fuel oil on the mysid shrimp Neomysis integer. Mar. Ecol. Progress Series. 12: 29-41.

Magalhães, V.F. \& Pfeiffer, W.C. (1995), Arsenic concentration in sediments near a 
metallurgical plant (Sepetiba Bay, Rio de Janeiro, Brazil). J. of Geochemical Exploration, 52: 175-181.

Mc Kenney JR, C.L. \& Neff, J.M. (1981), The ontogeny of resistance adaptation and metabolic compensation to salinity and temperature by the caridean shrimp Palaemonetes pugio, and modification by sublethal zinc exposure. In: Biological monitoring of marine pollutants. Vernberg, F.J.; Calabrese, A; Thurberg, F.P. \& Vernberg, W.B. (eds.), Academic Press, pp.45-66.

Moore, M.N.; Widdows, J.; Cleary, J.J.; Pipe, R.K.; Salkeld, P.N.; Donkin, P.; Farrar, S.V.; Evans, S.V. \& Thomson, P.E. (1984), Responses of the mussel Mytilus edulis to copper and phenanthrene: interactive effects. Mar. Environ. Res., 14: 167-183.

Moraes, R.B.C. (1996), Efeitos de sedimentos contaminados em camarões marinhos. Tese (Doutorado) $\mathrm{IBCCF}^{\circ}$, Universidade Federal do Rio de Janeiro.

Moraes, R.B.C.; Correa JR, J.D. \& Allodi, S. (1994), Efeitos sub-letais do zinco em Penaeus brasiliensis : Estudos preliminares. In: Anais do III Simpósio de Ecossistemas da
Costa Brasileira. Publicação ACIESP $\mathrm{n}^{\circ}$ 87-1.

Naimo,T.J.; Atchison, G.J. \& Holland-Bartels, L.E. (1992), Effects of cadmium on physiological responses in the pocketbook mussel, Lampisilis ventricosa. Environ. Toxicol. and Chem., 11: 1013-1021.

Parsons, T.R.; Maita, Y. \& Lalli, C.M. (1984), A Manual of Chemical and Biological Methods for Seawater Analysis. Oxford: Pergamon Press, pp. 55-72.

Silva, O. (1977), Aspectos biológicos e pesqueiros de três espécies de camarões do gênero Penaeus nas costas do Estado do Rio de Janeiro e experimentos de cultivo. Tese (Mestrado) - Departamento de Zoologia Universidade Federal do Rio de Janeiro.

White, S.L. \& Raibow, P.S. (1982), Regulation and accumulation of copper, zinc and cadmium by the shrimp Palaemon elegans. Mar. Ecol. Progr. Series. 8: 95-101.

Received: September 10, 1997; Revised: November 07, 1997 ; Accepted: April 29, 1999. 